De Tampere à Séville : bilan de la sécurité européenne $(1 / 2)$

\title{
Une politique européenne commune sur l'immigration clandestine ? Sangatte et au-delà
}

A Common European Policy on Illegal Immigration? Sangatte and Beyond chercheurs de Cultures \& Conflits

\section{OpenEdition}

\section{Journals}

Édition électronique

URL : http://journals.openedition.org/conflits/785

DOI : $10.4000 /$ conflits.785

ISSN : $1777-5345$

Éditeur :

CCLS - Centre d'études sur les conflits lilberté et sécurité, L'Harmattan

Édition imprimée

Date de publication : 1 mars 2002

Pagination : 133-144

ISBN : 2-7475-3029-9

ISSN : 1157-996X

Référence électronique

chercheurs de Cultures \& Conflits, « Une politique européenne commune sur l'immigration clandestine ? Sangatte et au-delà », Cultures \& Conflits [En ligne], 45 I printemps 2002, mis en ligne le 22 mars 2006, consulté le 30 mars 2021. URL : http://journals.openedition.org/conflits/785 ; DOI : https:// doi.org/10.4000/conflits.785

Ce document a été généré automatiquement le 30 mars 2021

Creative Commons License 


\title{
Une politique européenne commune sur l'immigration clandestine? Sangatte et au-delà
}

\author{
A Common European Policy on Illegal Immigration? Sangatte and Beyond
}

chercheurs de Cultures \& Conflits

La politique européenne sur l'immigration clandestine, telle qu'elle émane de l'accord franco-britannique sur Sangatte et des déclarations des deux ministres de l'Intérieur sur l'immigration clandestine, est à la fois peu judicieuse au regard du contexte politique de montée en puissance des populismes de droite radicale dans divers pays, et elle est potentiellement dangereuse pour nos valeurs et nos règles de droit. Si cette politique se trouve confortée et avalisée au sommet de Séville, nous sommes en droit de nous interroger sur le tournant autoritaire que cette politique prend en confondant sécurité et mesures coercitives. Elle a des conséquences graves en matière de respect des droits tout en ayant une « efficacité zéro » à l'égard du but qu'elle se propose. Il faut donc en changer radicalement, même si depuis vingt ans on s'entête à vouloir la poursuivre.

Etiqueter les touristes du tiers-monde, migrants et demandeurs d'asile, de clandestins ou d'illégaux dès qu'ils cherchent à fuir un conflit ou à prolonger au-delà de trois mois un séjour dans l'Union pour trouver du travail, les considérer comme une menace publique sous prétexte qu'ils importeraient leurs problèmes avec eux, et les transformer de populations soumises à un risque en populations dangereuses, voire les considérer en bloc comme des criminels, ou des alliés du terrorisme international, ne peut apporter un socle rationnel ou même raisonnable pour élaborer une politique européenne globale sur l'espace migratoire et pour gérer les relations de l'Union avec les pays qui l'entourent dans un cadre de liberté et de respect des individus qui circulent.

Une intégration par la petite porte pour les pays d'Europe centrale et orientale dont on accepterait simplement les travailleurs qualifiés, accompagnée d'une politique de coercition renforcée à l'égard des pays du Sud, comme celle de l'instauration d'un 
contrôle des frontières maritimes par les marines nationales qui s'apparenterait à un " filet de sécurité » pour bloquer les flux de personnes fuyant des conflits internes, ravivera des tensions déjà existantes à l'échelle internationale. Il en va de même d'une politique de refus de visas Schengen toujours plus dure et des pressions et accords visant à dresser les gouvernements du Maghreb contre leur population en les poussant à criminaliser ceux qui émigrent sans autorisation. Ces politiques créent des points de fixation ou de rassemblement de populations aux frontières extérieures de l'Union comme Ceuta et Melilla provoquant le désespoir et parfois la mort de nombreuses personnes, créant ainsi de l'arbitraire sans à aucun moment être une "solution » à la régulation migratoire globale.

A l'intérieur de l'Union, un jeu de ping-pong de mauvaise foi pour "contenir", s'échanger ou se renvoyer "les clandestins les mieux connus des services de police" que sont les personnes échouées ou conduites par les autorités de polices elles-mêmes à Sangatte, n'aura guère plus de légitimité sur le long terme. Les contradictions nationales détournant, mais ne stoppant pas la circulation des personnes, débouchent sur la fabrication de "nasses » aux frontières intérieures de l'Union (mais extérieures de Schengen dont le Royaume-Uni ne fait pas partie, concernant la partie de l'accord sur la libre circulation) comme celle de Sangatte, dans le Pas de Calais, où échouent pêle-mêle des primo arrivants, des personnes refoulées, des demandeurs d'asile. La tentation des autorités d'appliquer des conditions juridiques " exceptionnelles » à ces lieux qui sont sur leur territoire aboutit paradoxalement à la constitution par les gouvernements de zones où ils refusent eux-mêmes leurs propres règles de droit quand elles profitent aux individus. En cela Sangatte n'est pas unique, les zones d'attente dans les aéroports internationaux, certains centres de rétention ou centres d'accueil fermés comme on les appelle ailleurs, mettent en danger les droits élémentaires de la personne en continuant à vouloir appliquer l'autorité de l'Etat à ces personnes tout en leur refusant les droits pleins et entiers reconnus à ceux qui vivent sur le territoire de cet Etat. La souveraineté, détachée de la territorialité et des droits qui lui sont liés, devient un simple "cache sexe " de l'arbitraire au quotidien qui règne dans ces lieux et qui explique l'hétérogénéité extrême des situations des populations, rappelant certaines descriptions de l'hôpital général au XVIIIème siècle où l'on entassait fous, malades et indigents. Dans ces zones de « droit inégal » où la police fait aussi la justice et la prison avec de moins en moins de contrôle sur ces activités, et parfois avec un sentiment des policiers d'être injustement abandonnés par leurs propres autorités politiques, les normes et valeurs qui font l'exemplarité de l'Union Européenne sont mises entre parenthèses.

De plus, cela met en danger, au cœur même de l'Union, le processus d'intégration et la cohésion sociale des sociétés multiculturelles qui composent l'Europe, et qui ont toujours été (que cela soit admis ou non par leurs gouvernants) des pays de migration, de circulation des personnes.

Cette politique de sécurisation et de criminalisation de l'immigration qui date du milieu des années 1980, un moment atténuée avec les déclarations de Tampere, revient en force avec la préparation du sommet de Séville et les études de faisabilité d'une mise en réseau des polices des frontières des pays de l'Union. Elle est le produit, avant même d'invoquer le 11 septembre, d'un affrontement entre une logique de mise en place de la libre circulation des personnes et de la création d'une identité citoyenne européenne pour tous, et une logique sécuritaire coercitive de "maitrise », de blocage des flux 
(internes comme externes) des populations débouchant sur la méfiance, le soupçon à l'égard des autres (y compris communautaires).

Non directement réductible à un affrontement entre premier pilier, techniques communautaires et troisième pilier, techniques intergouvernementales, cette lutte masquée par le soi-disant laboratoire Schengen transformé depuis en acquis, est soustendue par différents groupes de fonctionnaires et d'ONG à l'échelle transnationale et par leur influence sur et au sein des structures de l'Union (commission, conseil, parlement, cour). Elle oppose différents types de professionnels ayant des intérêts divergents qui font éclater les politiques gouvernementales nationales comme autant de fruits murs et recomposent les alliances et les oppositions selon cet axe : circulation des personnes comme un droit ou comme un danger.

La politique actuelle exacerbe la tension en multipliant les textes contradictoires et en donnant des armes juridiques à chaque camp. Elle est néanmoins induite de la coupure institutionnelle qui fait que l'on pense la migration au sein de groupes qui sont socialisés différemment, les uns mettant en avant les droits et leur nécessaire incrémentation et pour qui sécurité rime avec garantie juridique et que l'on retrouve surtout dans le premier pilier, alors que les autres groupes venant des second et troisième piliers, sont guidés par les préoccupations d'(in)sécurité de certains groupes sociaux souvent minoritaires en nombre mais bruyants, pouvant voter sur ce seul critère et par là relayés par des hommes politiques et certains médias. Ces préoccupations que l'on dit à tort légitimes sous prétexte qu'elles seraient majoritaires (ce qui reste justement à démonter) trouvent souvent leur seule cohérence à partir d'une focale sur les im-migrants non occidentaux et leurs enfants (même s'ils ont acquis la nationalité du pays où ils résident) afin de réunir en un seul « mal social » les divers problèmes de la violence politique, du crime, du chômage, de l'urbanisation.

En tant qu'analystes nous avons des responsabilités dans la mise en place de ces « ultra solutions " pour reprendre la formule de Watzlawick où l'entêtement à ne pas vouloir reconnaître que l'on a pu se tromper collectivement de méthode (ici l'intergouvernemental) et d'objectif central (la liberté plutôt que la maîtrise) a débouché sur la création de mythes tenaces visant à écrire l'histoire des migrants d'une certaine manière au lieu d'écrire celle de nos échecs. Il y a en effet au moins cinq façons d'analyser la question de l'immigration ou cinq récits qui se propagent et dont un seul a peut-être une chance de nous faire réfléchir autrement alors que les autres convergent sur les politiques actuelles : c'est à ce niveau des raisonnements ou des récits qu'il faut montrer les inconséquences des discours habituels et inventer une autre politique migratoire pour l'Europe.

Une autre politique migratoire pour l'Europe

L'immigration clandestine constitue un risque pour la stabilité et la cohésion sociales. Sans attenter à la sécurité quotidienne des individus ou même à celle de l'Etat, par sa simple présence, qu'elle soit légale ou non, elle modifie le rapport de forces au sein d'une société donnée pour dire ce que sont les valeurs de cette société. Elle transforme les identités collectives d'une nation. En revanche, elle a l'avantage, par ces normes différentes, de pallier momentanément à des difficultés, que celles-ci soient rapportées à un équilibre social à long terme prenant en compte les besoins du marché du travail ou aux changements démographiques liés aux taux de fécondité ou à l'allongement de la durée de la vie « inactive ». 
L'immigration clandestine constitue une menace criminelle transnationale, raison pour laquelle polices et justices doivent être engagées aux frontières et au-delà de ces dernières. Elle est avant tout affaire de police, d'ordre public, de volonté et de souveraineté.

L'immigration clandestine est une menace externe à la sécurité des Etats, raison pour laquelle les forces armées et les services de renseignements doivent être engagés pour protéger les frontières et les garder coûte que coûte. L'immigration non contrôlée est un risque au sens de la défense nationale car elle importe avec elle le «terreau » du terrorisme transnational et agit à la manière d'une cinquième colonne transformant des territoires républicains en « zones de non droit».

L'immigration dite illégale est un phénomène social à la croisée des problématiques des frontières et du droit. L'inadéquation entre les deux termes est patente et se retrouve dans certaines définitions caractérisant des personnes. De ce fait, la réponse à apporter - eu égard aux problèmes suscités par l'immigration - serait de mettre fin à cette inadéquation par un changement du droit en vigueur et un abandon de certaines certitudes bureaucratiques incrémentées depuis près de vingt ans.

Le seul cadre correct d'analyse autorisant le débat est selon nous le dernier. Les autres ont inévitablement des effets pervers qui travestissent les données migratoires en un discours sur les risques et les menaces liés à l'existence d'un problème appelé l'immigration et les immigrants.

Le fait d'appréhender l'immigration clandestine en termes de menace pour l'identité, la citoyenneté et la cohésion sociale tient à une erreur profonde de lecture des phénomènes culturels et d'identités collectives assimilés à des " corps", à des " objets durs et définis » une fois pour toutes, au lieu d'en reconnaître leur hétérogénéité et leur plasticité. Ce discours est en fait souvent lui-même le principal danger pour la cohésion sociale tant invoquée en essayant de désigner un groupe comme le responsable de tous les divers maux d'une société. Les résultats du premier tour des élections présidentielles en France ont surpris tout un chacun et nous ont offert une configuration jusqu'alors impensable: un candidat d'extrême-droite était admis à participer au second tour sur la base d'un programme présentant les immigrés, tant légaux que clandestins, comme une menace intolérable pour... l'identité, la citoyenneté et la cohésion sociale françaises. Les immigrés étaient considérés comme inassimilables à la France et comme venant tous du Tiers Monde. Or, ce discours, intellectuellement et factuellement insoutenable, résonne, un octave plus bas, dans de nombreux services et organisations en charge de dire ce qu'est l'immigration clandestine et les moyens de la combattre. Donner une quelconque crédibilité à ces approches, en adoptant une politique qui s'en inspire, ne contribuerait pas, loin s'en faut, à défendre les objectifs poursuivis par l'Union européenne. On ne prévient pas le racisme futur et sa généralisation en renforçant l'autoritarisme au présent contre les groupes que ce racisme a désigné comme cible.

Si l'immigration n'est pas une menace en soi, si elle peut avoir des avantages, en est-il de même de l'immigration clandestine ? L'idée de la violation de la loi est sacralisée dans le discours au point d'associer tout défaut de papiers en règle à une menace $d u$ crime transnational. Mais souvent les lois sont à ce point contradictoires et enchevêtrées qu'elles créent les illégalités et qu'on ne vérifie leur respect strict que pour certains faits et certaines catégories de personnes. Le faire pour certains migrants venus du Sud en les soupçonnant plus que d'autres, relève de la suspicion policière, de 
démarches proactives qui conditionnent qui est placé sous surveillance et qui ne l'est pas, qui apparaît dans les catégories pénales et qui y échappe. A ce propos, il apparaît clairement que la création d'un réseau des corps de polices des frontières au niveau européen lié directement ou non à Europol et considérant le contrôle aux frontières comme une partie intégrante de la prévention du crime, serait absolument contreproductive en multipliant les "nasses" comme Sangatte à toutes les frontières. Franchir les frontières n'est pas, faut-il le souligner, une activité criminelle. Pour l'immense majorité des personnes, franchir les frontières dans l'Union européenne, c'est exercer un droit. Les agences de police ont effectivement l'autorisation d'arrêter et de contrôler des individus sur la base d'une suspicion d'un crime, mais cela ne peut se faire avec la multiplication de zones de «droit inégal» où l'Etat l'emporte sur l'individu. Valider cette procédure dans le contrôle des frontières, engage à créer des pouvoirs quasi illimités d'arrestation et de poursuite et accroît le risque de voir se multiplier des pratiques arbitraires.

Considérer l'immigration clandestine comme une menace externe à la sécurité, de telle ampleur que l'intervention des forces armées s'avérerait nécessaire, a aussi de sérieux effets pervers. L'immigration clandestine ne peut être lue à travers la thématique de la cinquième colonne, de l'ennemi intérieur infiltré pour aider une puissance extérieure. Le raisonnement en bloc ne fait pas sens. On ne peut faire glisser la suspicion policière contre quelques individus vers un raisonnement collectif englobant un groupe contre qui se battre. L'armée n'a pas sa place, même - et surtout - après le 11 septembre, dans la surveillance et le contrôle internes car, par son intervention, elle change le statut de ce contrôle en le désindividualisant et en transformant les migrants, ou les petits délinquants, en ennemis de la nation. L'armée ne peut être tout au plus qu'un point d'appui pour des enquêtes policières. Nous avons ainsi appris que la compagnie Eurotunnel s'était assurée les services d'un général à la retraite de l'armée britannique pour conseiller l'entreprise sur la manière de contrôler l'entrée du tunnel en France et que Sangatte serait sous surveillance britannique. Voilà le résultat du contrôle zélé par les Britanniques des immigrants clandestins arrivant de France par cette voie. Dès lors, deux questions sont primordiales dans ce cadre d'analyse: d'abord la construction d'une expertise militaire en tant que solution; ensuite l'insuffisance d'un système transférant au secteur privé le contrôle des frontières. Il semble de moins en moins improbable par exemple qu'un département ministériel britannique demande conseil, même de façon informelle, au ministère de la défense - au nom de la lutte antiterroriste - sur la façon de protéger l'accès au territoire britannique à des demandeurs d'asile afghans parqués à Sangatte en France et suspectés de soutenir al-Qaida. Il est important de se rappeler que même si la question de l'immigration clandestine s'immisce dans le domaine militaire, ce n'est pas encore le cas de ses conséquences en termes de contrôle judiciaire. La guerre non déclarée du gouvernement britannique contre l'immigration clandestine a conduit à des procès qui ont amené la Cour d'Appel à entrer en conflit avec l'administration. Dans l'affaire International Transport Roth GmbH $\&$ ors ${ }^{1}$, la Cour d'Appel a estimé que les effets de la législation britannique imposant des sanctions aux petits transporteurs constituaient une violation de la Convention européenne des droits de l'Homme puisque la sanction ne respectait pas le principe de la proportionnalité. Au niveau de la Cour d'instance, le défi était encore plus problématique pour l'administration puisque le juge y a non seulement trouvé une violation de la Convention européenne des droits de l'Homme, mais aussi une violation du droit communautaire et du droit de libre circulation. 
Il nous faut donc revenir à la question centrale, une fois écartée ces approches autoritaires qui sont des « décisions absurdes " générant et amplifiant les problèmes au lieu de réguler la situation, mais dont les initiateurs s'entêtent à penser qu'il faut aller encore plus loin dans la même voie au lieu de changer de cap. Qui sont donc ces immigrés clandestins dont il faudrait encadrer les agissements parce qu'ils sont la menace extérieure et le support du crime transnational? Sont-ils les extracommunautaires non occidentaux dont les modes de vie, la religion, les identités nous mettraient en péril ? L'association mentale est si forte que la question est pour certains un pléonasme, et pourtant. Prenons l'exemple de l'Australie, seul pays dans le monde à avoir construit un appareil statistique très sophistiqué en termes de flux et à disposer d'une information aussi détaillée sur la population résidant illégalement sur son sol. Compte tenu des investigations substantielles lors des contrôles aux frontières et grâce au croisement des données relatives aux entrants et aux sortants ${ }^{2}$, les services australiens ont constaté d'une part que le franchissement illégal des frontières représente à peine $20 \%$ de l'immigration illégale, et que, malgré les rhétoriques gouvernementales contre les kurdes et les asiatiques, les clandestins sont avant tout des occidentaux (américains et anglais). D'après leurs données l'illégalité provient avant tout des personnes qui restent sur place au-delà de la période autorisée (approximativement 20000 personnes par an, soit aujourd'hui un total de 58748 personnes, suite à un accroissement de $11,8 \%$ en 2000) ; alors que ceux qui arrivent illégalement par voie aérienne (1 695 cas ont été recensés en 2000) ou par voie maritime (4 175 cas relevés en 2000) ne représentent que 6000 cas. Ainsi, la grande majorité des immigrés clandestins en Australie est composée de ceux qui demeurent sur le territoire australien au-delà de la durée de validité de leur visa.

Ces données concordent avec les estimations de la Commission européenne pour l'Union européenne en 1989 quant au ratio de personnes restant illégalement sur le territoire par rapport aux personnes entrant sur le territoire sans autorisation.

Or, qui sont ceux qui décident de demeurer au-delà de la période autorisée de leur visa acquis légalement? Ceux auxquels on ne fait pas attention, qui sont moins visibles. Selon le critère de nationalité, ils proviennent pour l'Australie en majorité de deux pays : le Royaume-Uni (avec le plus fort pourcentage) et les Etats-Unis. En ce qui concerne les arrivées non autorisées par voie aérienne, les Britanniques sont classés au sixième rang. C'est seulement pour ce qui est des arrivées non autorisées par voie maritime que les Britanniques ne figurent pas dans le « top $10 »$. En ce qui concerne le travail illégal en Australie, selon le site Internet du gouvernement, "des preuves anecdotiques montrent que la plupart des personnes qui dépassent la date de validité de leur visa, le font pour des raisons économiques surtout pour profiter des opportunités offertes par le marché de l'emploi. Les travailleurs illégaux sont généralement issus des mêmes pays que les personnes restant au-delà de la période autorisée ". Là aussi, la situation de l'Australie peut éclairer celle de la France, du Royaume-Uni et plus largement, de l'Union européenne. Comme nous l'avons vu, le groupe le plus important d'immigrés en situation irrégulière en Australie est composé de Britanniques. Ces personnes sont connues. Il s'agit de jeunes Britanniques en vacances prolongées en cette période charnière de leur vie où ils sont censés commencer des études universitaires ou se lancer dans le marché du travail : plutôt que de repartir, ils décident de rester pour travailler, sans permis, de façon temporaire et occupent souvent un emploi précaire. 
Si nous revenons au parallèle avec l'Union Européenne, malgré la rhétorique britannique ou française relative à la question de l'immigration clandestine qui serait le fait d'extra-communautaires non occidentaux, nous sommes devant une situation qui n'est pas si différente. La présidence portugaise en l'an 2000 avait d'ailleurs proposé un projet de Règlement autorisant la Communauté européenne à passer des traités avec des pays tiers (notamment avec les Etats-Unis) pour permettre aux ressortissants de ces Etats tiers de demeurer en Europe plus longtemps que les trois mois autorisés par les visas Schengen. En effet plusieurs de ces Etats tiers puissants s'inquiétaient de voir certains de leurs ressortissants devenir des clandestins (principalement parce qu'ils demeurent sur le territoire au-delà de la période autorisée) à cause des règles européennes en matière de séjour et voulaient faire cesser la situation. Le code de l'immigration britannique a ainsi une certaine flexibilité pour les jeunes gens issus du Commonwealth : il les autorise à vivre et à travailler au Royaume-Uni pour une période maximale de deux ans et leur évite de facto de se mettre en situation d'illégalité, même si cela n'est toujours pas possible pour les citoyens américains, malgré leurs demandes.

Aussi, la réponse apportée par les Etats européens soumis au risque de l'immigration clandestine - conséquence d'une expiration des visas-, est de modifier les lois sans criminaliser ces « étrangers »-là. Ils peuvent rester plus de trois mois sur place. C'est une manière d'appréhender et de traiter l'immigration clandestine contenue dans le dernier cadre d'analyse présenté : désacraliser la loi, loi qui n'est pas immuable et l'adapter aux circonstances. Celui-ci a donc déjà cours au sein de l'Union européenne. Les règles de la Communauté européenne relatives à la libre circulation des personnes diront bientôt que les ressortissants d'Europe centrale et orientale ne seront plus des immigrés clandestins à l'intérieur des frontières de l'Union. Nous aurons ainsi diminué d'un coup et de façon très importante la population clandestine sans pour autant militariser nos frontières, ni qualifier les migrations de crime transnational et sans construire d'autres catégories constituant de nouvelles menaces à notre identité.

On le voit, si l'on tient compte du fait que la majorité des étrangers qui de factocontreviennent aux lois de l'immigration (et qui ne sont pas nécessairement poursuivis pour ces infractions) sont de jeunes ressortissants de pays avec lesquels nous avons des relations amicales, le type de mesures proposées par les gouvernements français et britannique contre les clandestins et leur arrivée, est clairement inapproprié. Il faudrait demander à ces gouvernements d'expliquer leurs propositions et leur demander si la cible de leur action est la lutte contre l'immigration clandestine ou une guerre sans nom aux pays du Sud et à leurs ressortissants? Il se pourrait bien, si l'on avait le même appareillage statistique que l'Australie, que nous "découvrions" que la majorité des étrangers clandestins sont issus de pays avec lesquels les Etats européens ont des relations amicales comme l'Amérique du Nord et avec lesquels ils ne tiennent pas à prendre des mesures restrictives. La question de l'immigration clandestine est empreinte d'une réelle émotivité. Jusqu'à présent, elle a été traitée de façon émotionnelle voire irrationnelle. L'invisibilité sociale de ceux qui résident en Europe plus longtemps que prévu mais en repartent aussi après un certain temps contraste avec la visibilité sociale forte de ceux qui franchissent illégalement les frontières et sur lesquels les médias focalisent. A un moment où un autre, les gouvernements, en quête d'une politique honnête et cohérente, devront modifier leur discours politique vis-à-vis des clandestins ou prendre des mesures visant à identifier clairement cet ennemi « invisible » auquel ils font sans cesse allusion. Les politiques devront toutefois être extrêmement prudentes pour ne pas discriminer certains 
clandestins par rapport à d'autres. Car si leurs propositions devaient être contraires aux lois de l'Union européenne réprimant le racisme, il faudrait alors réévaluer le texte en conséquence.

\section{NOTES}

1.. The Times, 26 février 2002

2. . www.immi.gov.au/illegals/border2000/

Aussi, selon un article paru dans le Financial Times du 15 mai 2002, le budget australien vient d'accuser un déficit surprise en raison d'une hausse des dépenses relatives à la sécurité des frontières.

\section{RÉSUMÉS}

European policies concerning illegal immigration are criticised in light of France and GB's agreement about Sangatte (a camp for illegal immigrants). Analytical frameworks that see illegal immigration as a cultural, criminal, or national security problem are contested. A more rational approach would be to consider who the illegal immigrants are, before making immigration laws. Australian immigration figures - analogous to those of European nations - reveal that typical illegal immigrants are young British or US citizens who have overstayed their visas in order to work or to enrol in a university ; they are not criminals. Rather than being driven by emotion, immigration laws should be written to account for the realities of immigration.

\section{INDEX}

Index géographique : Union européenne

Mots-clés : construction européenne, immigration 